\title{
Editorial
}

\section{The Price of a Surgical-Site Infection: More Than Just Excess Length of Stay}

\author{
Edward S. Wong, MD
}

In this issue of Infection Control and Hospital Epidemiology, Kirkland et al report their study of the impact of surgical-site infections (SSIs) by the matched cohort method, comparing patients with SSIs to control patients of approximately the same age who have undergone the same operative procedure during the same time period. ${ }^{1}$ Controls also were matched for the same National Nosocomial Infection Surveillance (NNIS) Study index and even the same surgeon, if possible. The study found that SSIs prolonged hospital stay by a median of 6.5 days and incurred an attributable direct cost of $\$ 3,089$. For infection control practitioners, these results are reassuring, because they serve to justify our professional existence; for healthcare administrators, they are equally reassuring, because they justify the resources and expenditures invested in infection control programs. However, the findings are hardly unique. Multiple prior studies dating from the 1950 s through the 1980s have shown that SSIs are associated with excess hospital stay and excess cost. ${ }^{2-6}$ In these studies, the magnitude of the excess stays has varied from 1.3 to 23.8 days. The variations could be explained on the basis of the different surgical populations being evaluated, the different data-collection methods used, as well as the different criteria for matching infected patients to noninfected controls.

As pointed out by Kirkland et al, the majority of those studies were done in the 1970 s and early 1980 s, prior to the onset of hospital reimbursement by diagnosis-related groups (DRGs). In the current era, in which DRG and managed-care rule, the lengths of hospital stay for most admissions have decreased. Due to the pressure to minimize hospital stays, one almost can predict a priori that the excess hospital stay attributable to SSIs would be lower in the current era, and indeed the median excess length of stay of 6.5 days found in Kirkland et al's study is roughly half of what Green and
Wenzel's study found in $\mathbf{1 9 7 6}$ prior to the advent of prospective reimbursement. ${ }^{6}$

There may be yet another explanation for the shorter excess stay attributable to SSIs. In the matched-cohort method, the object is to achieve as close a match as possible between patients who do and do not develop SSIs in order to minimize confounding variables that independently predispose to long hospitalization, as well as to wound infections. In prior studies, various criteria have been used for matching: age, operative procedure, admitting or discharge diagnoses, the number and severity of associated diseases, and nutritional status. In Kirkland et al's study, control patients were matched by commonly used variables, including age, operative procedure, and operating surgeon. Several novel criteria also were used, such as the NNIS risk index and the American Society of Anesthesiologists' score. These indices or scores are generally used to stratify operative patients in regard to their risk for subsequent infection. ${ }^{7}$ These variables were used in this study presumably as proxies for underlying severity of illness. While their use for this purpose is logical and reasonable, it remains unproven. Nonetheless, the set of criteria used by Kirkland et al for matching appear to be fairly stringent and would likely result in patient groups that are closely matched. As all epidemiologists know, the closer the match, the smaller the difference between groups. Thus, the excess length of stay may have diminished on this basis.

The price you pay for stringent matching criteria is that a greater number of patients with SSIs (cases) might not be able to be matched with controls, which in turn could lead to selection biases. In most studies, depending on the matching criteria, $10 \%$ to $20 \%$ of infected patients are generally not matched to controls., ${ }^{2,4,6}$ I am pleasantly surprised that, in the study by Kirkland et al, only 17 (6\%) of

From the Infectious Disease Section, Department of Medicine, McGuire Veterans' Affairs Hospital, and the Department of Medicine, Medical College of Vinginia, Vinginia Commonwealth University, Richmond, Virginia. 
272 patients who developed SSI could not be matched to uninfected controls. Perhaps that is a reflection of the large database of over 22,000 patients with surgical procedures available to the authors for matching.

It should be noted that the matched-cohort comparison is not the only method for estimating excess hospital stays and costs associated with nosocomial infections. Physicians or other trained personnel can be used to assess the need for continued hospitalization. ${ }^{5}$ In this method, physicians would concurrently review the medical record and other relevant clinical information to decide whether to attribute each day of hospitalization to the primary reason for hospitalization or to the nosocomial infection. A more standardized variation of this direct method is the Appropriateness Evaluation Protocol, developed by Gertman and Restuccia, in which explicit criteria are used to assign hospital days as non-infection-related or infection (SSI)-related..$^{8,9}$ These direct methods generally yield lower excess hospital stays and excess cost when compared to matched-cohort methods. Which resultwhether it is the higher estimate generated by matchedcohort method or the lower one produced by direct review-more accurately reflects the true cost of SSI is not known. What is known is that direct-review methods are more labor-intensive and time-consuming, and thus generally not done.

Matched-cohort comparison does offer one important advantage over direct-assessment techniques. By comparing differences in death rates in patients with and without infection, one can determine an attributable mortality associated with that infection. In Kirkland et al's study, 20 (7.8\%) of patients with SSIs died, compared to $9(3.5 \%)$ of matched surgical patients without infections. These results allowed the authors to calculate an attributable mortality due to SSIs of $4.3 \%$ (7.8\% minus $3.5 \%$ ) and relative risk of death of 2.2 .

On the basis of recent studies on postdischarge surveillance for SSIs, we now know that SSIs often do not become manifest until after patients are discharged from the hospital. ${ }^{10,11}$ Previous cost studies on SSIs that relied solely on inpatient surveillance would have missed these infections and as a result would underestimate the overall cost of SSIs. To their credit, the study by Kirkland et al did attempt to capture additional cost associated with subsequent rehospitalization. They found that a whopping 94 infected patients, or $41 \%$ of the 229 who survived the original hospitalization, required rehospitalization within 30 days of discharge, compared to 17 (7.4\%) of controls without infections. The authors conceded that not all SSIs that developed after discharge require rehospitalization and that the cost of mild to moderate SSIs that could be managed as outpatients in clinics and offices were not captured by their study. Nonetheless, when the impact of readmissions alone were factored in, the excess length of stay jumped from 6.5 days to 12 days and the cost from $\$ 3,089$ to $\$ 5,038$ per episode of infection. The almost doubling of excess stay and cost with the inclusion of postdischarge events serves to point out how myopic previous studies were when they focused purely on outcomes occurring during the original hospitalization.

The process of solving any problem necessarily begins with the recognition of the magnitude and extent of the problem. We owe thanks to Kirkland and coworkers for providing us a more complete picture of the price we pay for SSIs. This broader picture of the cost associated with SSIs is especially important in today's healthcare environment, in which many patient-care services, including the care of adverse outcomes such as SSIs, are being shifted toward the outpatient arena and thus potentially hidden. Recognition, however, is only part of the process. We must continue to find and evaluate interventions that will either prevent or reduce the number of SSIs. Some measures for the prevention of SSIs have stood the test of time and evidence-based medicine. The use of preoperative prophylactic antibiotics is one such measure. ${ }^{12,13}$ Ensuring the appropriate use and timing of preoperative antibiotics has been shown to be efficacious and should be the cornerstone of any good SSI prevention program. Other measures are not as well-established. Feedback of risk-adjusted and procedure-specific SSI rates to surgeons is cited by the authors as having brought their infection rate down from $1.6 \%$ to $1 \%$ over a 2 -year period. Feedback of SSI rates has not been shown definitively to be efficacious and has been given a category IB rating by the Centers for Disease Control and Prevention in their recently published "Guideline for Prevention of SurgicalSite Infection, 1999." 14 In the CDC's ranking scheme, category IB interventions are backed by some studies as being efficacious but lacking support by definitive, well-designed clinical studies. Finally, there are measures that are just entering the testing stage. Exciting are preliminary studies that suggest nares colonization with Staphylococcus aureus predisposes toward SSIs and that depopulation with antibiotic ointment will reduce that risk. ${ }^{15,16}$ I refer interested readers to the $\mathrm{CDC}$ guidelines and other reviews for up-to-date discussions of practices and interventions that have an impact on the risk of SSIs. ${ }^{14,17,18}$

\section{REFERENCES}

1. Kirkland KB, Briggs JP, Trivette SL, Wilkinson WE, Sexton DJ. The impact of surgical site infections in the 1990s: attributable mortality, excess length of hospitalization, and extra costs. Infect Control Hosp Epidemiol 1999;20:725-730.

2. Clark KSR Sepsis in surgical wounds, with particular reference to Staphylococcus aureus. Br J Surg 1957;44:592-596.

3. Committee on Control of Surgical Infections of the Committee on Pre and Post-operative Care, American College of Surgeons. Manual on Control of Infection in Surgical Patients. Philadelphia, PA: J.B. Lippincott Co; 1976:11-14.

4. Freeman J, Rosner BA, McGowan JE Jr. Adverse effects of nosocomial infection. I Infect Dis 1979;140:732-740.

5. Haley RW, Schaberg DR, Von Allmen SD, McGowan JE Jr. Estimating the extra charges and prolongation of hospitalization due to nosocomial infections: a comparison of methods. J Infect Dis 1980;141:248-257.

6. Green JW, Wenzel RP. Postoperative wound infection: a controlled study of the increased duration of hospital stay and direct cost of hospitalization. Ann Surg 1977;185:264-268.

7. Culver DH, Horan TC, Gaynes RP, Martone WJ, Jarvis WR, Emori TG, et al. Surgical wound infection rates by wound class, operative procedure, and patient risk index. Am J Med 1991;91(suppl 3B):152S-157S.

8. Gertman PM, Restuccia JD. The Appropriateness Evaluation Protocol: a technique for assessing unnecessary days of hospital care. Med Care $1981 ; 19: 855-871$. 
9. Wakefield DS, Pfaller MA, Hammons GT, Massanari RM. Use of the Appropriateness Evaluation Protocol for estimating the incremental costs associated with nosocomial infections. Med Care 1987;25:481488.

10. Brown RB, Bradley S, Opitz E, Cipriani D, Pieczarka R, Sands M. Surgical wound infections documented after hospital discharge. $A m J$ Infect Control 1987;15:54-58.

11. Weigelt JA, Dryer D, Haley RW. The necessity and efficiency of wound surveillance after discharge. Arch Surg 1992;127:77-81.

12. Platt R, Zaleznik DF, Hopkins CC, Dellinger EP, Karchmer AW, Bryan $\mathrm{CS}$, et al. Perioperative antibiotic prophylaxis for herniorrhaphy and breast surgery. $N$ Engl J Med 1990;322:153-160.

13. Classen DC, Evans RS, Pestotnik SL, Horn SD, Menlove RL, Burke JP. The timing of prophylactic administration of antibiotics and the risk of surgical-wound infection. N Engl J Med 1992;326:281-286.

14. Mangram AJ, Horan TC, Pearson ML, Silver LC, Jarvis WR, the Hospital
Infection Control Practices Advisory Committee. Guideline for prevention of surgical-site infection, 1999. Infect Control Hosp Epidemiol $1999 ; 20: 247-280$.

15. Wenzel RP, Perl TM. The significance of nasal carriage of Staphylococcus aureus and the incidence of postoperative wound infection. J Hosp Infect 1995;31:13-24.

16. Kluytmans JA, Mouton JW, VandenBergh MF, Manders MJ, Maat AP, Wagenvoort $\mathrm{JH}$, et al. Reduction of surgical-site infections in cardiothoracic surgery by elimination of nasal carriage of Staphylococcus aureus. Infect Control Hosp Epidemiol 1996;17:780-785.

17. Wong ES. Surgical site infections. In: Mayhall CG, ed. Hospital Epidemiology and Infection Control. Baltimore, MD: Williams \& Wilkins, Inc; 1996.

18. Kluytmans JAJW. Surgical infections including burns. In: Wenzel RP, ed. Prevention and Control of Nosocomial Infections. Baltimore, MD: Williams \& Wilkins, Inc; 1997. 